\title{
EDITORIAL
}

\section{Pneumococcal conjugate vaccines: New hope for infancy}

\author{
VACUNAS ANTI NEUMOCÓCCICAS CONJUGADAS: \\ NUEVAS ESPERANZAS PARA LA INFANCIA
}

As discussed in the articles in this review, the pneumococcus is a major pathogen in young children. This bacteria has evolved to cause serious diseases including meningitis, pneumonia and sepsis as well as less serious illnesses such as otitis media and sinusitis. More recently the organism has successfully evolved both intermediate and high level resistance to one or more antibiotics as well. Although a polysaccharide vaccine has been available for prevention of disease in adults for some time, this vaccine is largely ineffective in the prevention of disease in children under two years of age. Unfortunately, it is these children who are at the highest risk of invasive pneumococcal disease, pneumonia and meningitis. In addition, WHO estimates that globally approximately 4 million children die each year of lower respiratory tract infection largely in the first year of life. Of this total, more than one million deaths are due to pneumococcal infection. Thus, the availability of a vaccine which is effective in young children offers the potential to prevent infections which are increasingly difficult to treat due to antibiotic resistance and to tremendously reduce infant mortality worldwide. In this issue, Dr. Ruvinsky has reviewed the epidemiologic burden of pneumococcal disease in his article. He points out that the burden of morbidity and mortality of the pneumoccocus is disproportionately borne by children in the developing world. In addition, antibiotic resistance levels have increased dramatically over the past decade with an estimated $28 \%$ of isolates now resistant to penicillin with resistance levels tending to be higher in children less than two years of age.

A seven valent pneumococcal conjugate vaccine has recently been licensed in the United States and Europe for use in children beginning at two months of age. In pre-licensure studies of this vaccine, it was more than $95 \%$ effective in reducing the risk of invasive disease due to vaccine serotypes and resulted in an almost $90 \%$ reduction in the overall burden of disease regardless of serotype. Ongoing studies have demonstrated that this level of efficacy is maintained with time and that an increase in the risk of invasive disease due to non-vaccine serotypes (replacement) has not occurred.

Although there are more than 90 serotypes of pneumococci and the serotype distribution of types causing invasive disease in children varies in different countries of the world, the seven serotypes in this vaccine account for $85 \%$ of the disease in children in the USA and the majority invasive disease in most countries. In this issue, Dr. Ruvinsky points out that within Latin America, the serotype specific coverage level is $61 \%$ in children less than two years of age. To attain the coverage levels attained by a seven valent vaccine in the USA, a nine valent vaccine will be required in Latin America. It is important to note that in all countries of the world, antibiotic resistance is almost exclusively limited to the seven serotypes in the currently available conjugate vaccine.

In considering the inclusion of new vaccines into a vaccine program, it is important to understand the potentially preventable disease burden. As Dr. Lagos points out in this issue, studies of pneumococcal 
disease in children tend to underestimate this burden. True rates of bacteremia are underestimated because cultures may not be obtained prior to institution of antibiotics in many cases. In addition, very few pneumococcal respiratory infections in children are associated with sputum to culture or positive blood cultures. Hence the contribution of the pneumococcus to these diseases has been underestimated. In our pre-licensure evaluation of the pneumococcal conjugate vaccine, we estimated the efficacy of this vaccine for pneumonia in children. We observed a $11 \%$ reduction in all pneumonia cases and a $33 \%$ reduction in pneumonias with any abnormality on $x$-ray. These data would indicate that the pneumococcus was accounting for a greater proportion of all pneumonia and $x$-ray positive pneumonia than we would have estimated prior to the vaccine study. With the licensure and use of the seven valent conjugate vaccine in various countries of the world it will be important to reevaluate the impact of vaccination on pneumonia using the vaccine itself as a probe.

In addition to studies evaluating the effect of the introduction of pneumococcal conjugate on disease burden and disease epidemiology, it will be important to maintain serotype specific disease surveillance in all areas of the world where vaccine is utilized. As pointed out by Drs. Maria Hortal and Teresa Camou in this issue, the molecular epidemiology of the pneumococcus in complicated. Capsular transformation of one serotype to another has been observed in the laboratory with the pneumococcus. With the selective pressure applied by the conjugate vaccine to the serotypes included in the vaccine, surveillance for capsular transformation in the field with consequent escape from vaccine protection will be critical.

In conclusion, the articles in this issue stress the importance of the pneumcoccus as a major cause of morbidity and mortality in children worldwide. After many years of research and development, we now have a seven valent conjugate vaccine available which is highly effective in preventing invasive disease in young children. Although optimal coverage in many countries in Latin America will require a nine or eleven valent vaccine, more than half of the total disease burden and almost all antibiotic resistant infections are preventable using the currently available vaccine. With introduction of pneumococcal conjugate vaccine, disease epidemiology and serologic surveillance will be important to assure maximal public health impact.

Steven B. Black, MD and Henry R. Shinefield MD

Co-Directors, Kaiser Permanente Vaccine Study Center

Oakland, California, USA 